\title{
Novel Ion-sensitive Field-effect Transistor with Ion Sensing Polymerized Silsesquioxane Membrane on Detachable Metal Disk
}

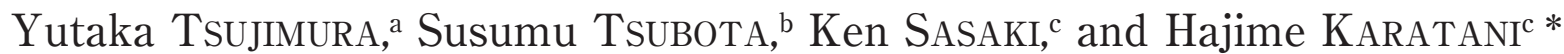

\begin{abstract}
aNational Institute of Advanced Industrial Science and Technology Kansai (Midorigaoka, Ikeda, Osaka 563-8577, Japan)

Present address: Nagase ChemteX Corporation (236, Tatsunocho-nakai, Tatsuno, Hyogo, 679-4124, Japan)

${ }^{b}$ National Institute of Advanced Industrial Science and Technology Kansai (Midorigaoka, Ikeda, Osaka 563-8577, Japan)

'Department of Biomolecular Engineering, Graduate School of Science and Technology, Kyoto Institute of Technology (Matsugasaki, Sakyo-ku, Kyoto 606-8585, Japan)
\end{abstract}

Received February 1, 2007 ; Accepted April 5, 2007

\begin{abstract}
We developed a new type of an ion-sensitive field-effect transistor (ISFET) with an ion sensing polymerized silsesquioxane (polySQ) membrane carrying a quaternary ammonium salt (QAS) as an ionophore. A noticeable feature of this ISFET is that the metal disc part is designed to be detachable from an FET body. Owing to this structure, the full polymerization of SQ at relatively high temperature became possible; leading to preparation of a durable ion sensing polySQ membrane on a metal disk connected to the FET gate terminal. The ISFET with the polySQ membrane carrying QAS (polySQ-QAS) (optimal content of QAS, $40 \mathrm{wt} \%$ in the matrix), prepared at $150^{\circ} \mathrm{C}$ for $3 \mathrm{~h}$, showed not only the theoretical potential response to the nitrate ion concentration in the range of $1.0 \times 10^{-6}$ to $0.10 \mathrm{~mol} \mathrm{dm}^{-3}(\mathrm{M})$ with the Nernst slop of $-61.5 \mathrm{mV} \cdot \mathrm{decade}^{-1}$, but also the rapid response $(3 \mathrm{~s}$ for $90 \%$ of response). Furthermore, the ion sensing property is favorably sustained even after being soaked in methanol for $24 \mathrm{~h}$.
\end{abstract}

Key Words : Detachable Ion-sensing Head, Polysilsesquioxane, ISFET

\section{Introduction}

It has been reported that silsesquioxane oligomer (referred to as SQ) (Fig. 1) is a promising material to prepare a glass-like membrane. ${ }^{1)}$

Furthermore, SQ can be easily mixed with other materials to be homogeneity and then polymerized to polysilsesquioxane (polySQ). Based on this property, it is expected that polySQ membranes with special functions can be obtained by incorporating functional compounds into the membranes.

By making use of these properties, we have developed an ionophore incorporated polySQ coated ion-sensitive field-effect transistor (ISFET). ${ }^{2)}$ In our previous study, to prepare the ion sensing polySQ membrane, a mixture of SQ and ionophores was cast on a metal disk of FET, and then allowed to polymerize at temperatures lower than

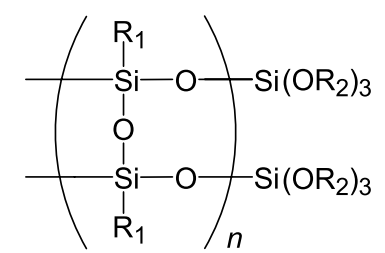

$\mathrm{R}_{1}=\mathrm{CH}_{3}$ or $\mathrm{C}_{6} \mathrm{H}_{5}, \mathrm{CH}_{3} / \mathrm{C}_{6} \mathrm{H}_{5}=2 / 1$

$\mathrm{R}_{2}=\mathrm{C}_{2} \mathrm{H}_{5}$ or $\mathrm{H}, \quad n \stackrel{\circ}{\doteqdot} 30$

Fig. 1 Molecular structure of silsesquioxane (SQ) oligomer $(n \doteqdot 30)$.
$100{ }^{\circ} \mathrm{C}$. Since relatively high temperatures, such as $150^{\circ} \mathrm{C}$, is required to fully polymerize $\mathrm{SQ}^{,-6)}$ a polySQ membrane on FET, prepared at $100^{\circ} \mathrm{C}$ or below, has suffered from lack of durability for ion sensing membrane. The fact that the performance of FET is deteriorated at temperatures higher than $125{ }^{\circ} \mathrm{C}$ has prevented to obtain a durable ion sensing polySQ membrane on FET.

In this study, the structure of the ISFET was devised to make its metal disk removable from the gate terminal part of FET. Based on this structure, a fully polymerized SQ membrane carrying an ionophore on the metal disk was successfully obtained; i.e., the full polymerization of $\mathrm{SQ}$ at desirable temperatures became possible on the detached metal disc.

We describe here 1) the structure of detachable ionsensing head, 2) the preparation of polySQ membrane carrying QAS, 3) the $\mathrm{NO}_{3}$ - sensing property, and 4) effect of polymerization temperature on the durability of the ion sensing membrane.

\section{1 Preparation of ISFET}

\section{Experimental}

A commercial metal oxide semiconductor MOSFET (2SK241) chip was employed to construct an ISFET.-8) The assembled ISFET is shown in Fig. 2. A titanium stick (7-mm in diameter, 6-mm long) connected to a gate terminal of the MOSFET was stuck to one end of the glass tubing $(7-\mathrm{mm}$ in external diameter, $4-\mathrm{mm}$ in internal diameter, 100-mm long) with a silicone-type adhesive. 


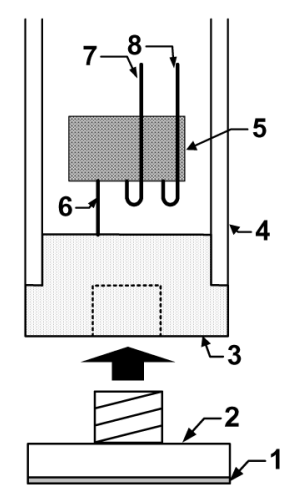

Fig. 2 Schematic drawing of the ISFET with a detachable ion sensing head. (1) ion sensing membrane, (2) metal disk gate with a screw, (3) titanium stick with a threaded hole, (4) glass tubing, (5) FET body, (6) gate terminal, (7) source terminal, (8) drain terminal.

In the titanium stick, a threaded hole (2-mm in diameter, 3-mm long) was also drilled on the opposite side to the gate terminal of the MOSFET. A metal disk (copper, titanium or stainless steel) to prepare the ion-sensing membranes ( $7-\mathrm{mm}$ in diameter, $2-\mathrm{mm}$ in thickness) equipped with a screw (2-mm in diameter, 3-mm long) can be fixed at the threaded hole of the titanium stick.

\section{2 Materials}

Glass Resin ${ }^{\circledR}$ (GR-100; Techneglas) was used as SQ to prepare the polySQ. ${ }^{2)}$ Dimethyloctadecyl-3-trimethoxyl silylpropylammonium chloride as a QAS was obtained from Shin-Etsu Silicone. Sodium nitrate $\left(\mathrm{NaNO}_{3}\right)$ was of guaranteed reagent grade. Sample solutions were prepared with deionized water.

\section{3 Preparation for ion-sensing membrane}

The ion-sensing membrane was prepared as follows. Both SQ (60 mg) and QAS (40 mg) were dissolved in 2 $\mathrm{mL}$ of 2-propanol. This 2-propanol solution is referred to as solution P. A $25-\mu \mathrm{L}$ aliquot of the solution $\mathrm{P}$ was cast on the metal disk and allowed to stand at the room temperature for $24 \mathrm{~h}$ to evaporate 2-propanol. The mixture of SQ and QAS free from 2-propanol is referred to as SQQAS. The metal disk coated with SQ-QAS was put in the oven kept at 120,150 or $180^{\circ} \mathrm{C}$ for $3 \mathrm{~h}$ to cure the membrane, resulting in polymerized SQ carrying QAS, referred to as polySQ-QAS. The prepared membrane was determined to be approximately $20 \mu \mathrm{m}$ in thickness by the microscope observation. The assembled ISFET was evaluated as a nitrate ion $\left(\mathrm{NO}_{3}{ }^{-}\right)$sensor.

Infrared spectra of the polySQ were measured by Perkin-Elmer 2000 spectrometer. Transmission measurements were made on sodium chloride plates. In this case, a $500-\mu \mathrm{L}$ aliquot of the solution $\mathrm{P}$ was cast on a slide glass, and then allowed to stand at room temperature for $24 \mathrm{~h}$ to evaporate 2-propanol. Then the slide glass was put in the oven kept at 120 or $150{ }^{\circ} \mathrm{C}$ for $3 \mathrm{~h}$. The prepared membrane was carefully peeled from slide glass with a spatula and adhered to the $\mathrm{NaCl}$ plate.

\section{4 Measurements}

The potential measurement was carried out at $25^{\circ} \mathrm{C}$ with an ISFET/pH meter (BAS). The potential was mon- itored with respect to a double-junction-type $\mathrm{Ag} / \mathrm{AgCl}$ reference electrode with $3 \mathrm{M} \mathrm{KCl}$ as an internal solution and $1 \mathrm{M} \mathrm{CH} \mathrm{CHOOLi}_{3} \mathrm{Cs}$ an external solution. The applied voltage and the current between the source and the drain were $5 \mathrm{~V}$ and $400 \mu \mathrm{A}$.

A $10 \mathrm{~mL}$ of test solution was agitated at a constant rotation speed using a magnetic stirrer during measurement. The concentration of each test solution was successively changed by injection of a 100 times higher concentration of the $\mathrm{NaNO}_{3}$ solution, usually $0.50 \mathrm{~mL}$. The activity coefficient of anion $(\gamma)$ was calculated, according to the Davies equation, ${ }^{9}$

$$
\log \gamma=-0.511 \cdot(I)^{1 / 2} /\left[1+0.33 \cdot \alpha \cdot(I)^{1 / 2}\right]-0.10 \cdot I
$$

where $I$ is an ionic strength and $\alpha$ is an ion size parameter.

\section{1 Properties of the membrane}

\section{Results and Discussion}

From the viewpoint of reaction mechanism, the polySQ membrane is obtained by the condensation of $\mathrm{SQ}$; i.e., the elimination of either the hydroxy group or alkoxy group of $\mathrm{SQ}$ is responsible for the formation of polySQ structure. Therefore, thermal treatment of SQ, called curing, is an important process to prepare a durable polySQ. It is also reported that hydroxy and/or alkoxy groups of SQ still remain in the polySQ prepared relatively at temperatures lower than $100{ }^{\circ} \mathrm{C} .{ }^{1)}$

First, we evaluated the adhesive property of the polySQ-QAS membrane toward three kind of metal disks; copper, titanium and stainless steel. From the evaluation, it was shown that the adhesion of polySQ-QAS to a stainless steel disk was the strongest and tightest of the three. Hence, the stainless steel was employed as the FET metal disk in this study. Regarding the mixing ratio of QAS to SQ, it was confirmed that the optimal ion sensing property was obtained at $40 \mathrm{wt} \%$ of QAS, as reported previously.2)

Subsequently, a polySQ-QAS membrane, prepared at room temperature, was cured at 120,150 and $180{ }^{\circ} \mathrm{C}$ for $3 \mathrm{~h}$. Unexpectedly, a polySQ-QAS membrane, cured at $180^{\circ} \mathrm{C}$, came off soon. This possibly indicates that the rapid curing causes insufficient adhesion between the polySQ-QAS membrane and the metal disk. By contrast, the polySQ-QAS membrane was found to be tightly prepared on the metal disk at both curing temperatures of 120 and $150{ }^{\circ} \mathrm{C}$.

\section{2 Evaluation of performance of ISFET}

Figure 3 shows plots of the potential response against the concentration of $\mathrm{NO}_{3}{ }^{-}$for the ISFET with a polySQQAS membrane prepared via the curing process at $150{ }^{\circ} \mathrm{C}$ for $3 \mathrm{~h}$. As shown, the linear potential response (curve a) against the $\mathrm{NO}_{3}$ concentration in the range of $1.0 \times 10^{-6}$ to $0.10 \mathrm{M}$ with a slope of $-61.5 \mathrm{mV} \cdot$ decade $^{-1}$ was obtained. This response was in good agreement with the theoretical Nernst response $\left(-59.2 \mathrm{mV} \cdot\right.$ decade $^{-1}$ at 298.15 $\mathrm{K})$. The time required to reach $90 \%$ response $\left(t_{90}\right)$ was approximately $3 \mathrm{~s}$ in the tested concentration range.

The durability of the ion sensing membrane was eval- 


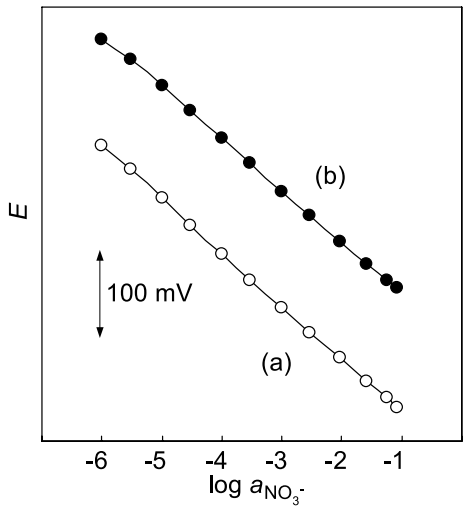

Fig. 3 Plots of potential response versus activity of $\mathrm{NO}_{3}{ }^{-}$for the ISFET with polySQ-QAS membrane cured at $150{ }^{\circ} \mathrm{C}$ for 3 h. Curves (a) and (b), before and after being soaked in methanol for $24 \mathrm{~h}$.

uated by the potential response property before and after being soaked in methanol. Even after the metal disk coated with polySQ-QAS was soaked in methanol for $24 \mathrm{~h}$, the Nernst slope was maintained at $-59.3 \mathrm{mV}$. decade $^{-1}$ (curve b) and no change in $t_{90}$ was present. The results indicate that the polySQ-QAS membrane prepared at $150^{\circ} \mathrm{C}$ has no leakage of QAS. By contrast, the ISFET coated with polySQ-QAS prepared via the curing process at $120^{\circ} \mathrm{C}$ no longer showed the potential response after being soaked in methanol for $24 \mathrm{~h}$. In relation to this, it was observed that the polySQ-QAS membrane was deteriorated after being soaked in methanol.

\section{3 IR evaluation of curing temperature}

FTIR spectra of polySQ-QAS membranes prepared on slide glass in the presence and absence of the curing process are shown in Fig. 4.

The spectra of SQ-QAS membranes before and after curing at $120{ }^{\circ} \mathrm{C}$ for $3 \mathrm{~h}$ (Fig. 4, curves (a) and (b)) exhibited typical stretching adsorption band of the $\mathrm{Si}-\mathrm{OH}$ at about $3200 \mathrm{~cm}^{-1}$. It should be noted that there is no substantial difference in the peak-height around $3200 \mathrm{~cm}^{-1}$ between the two curves, (a) and (b). This possibly indicates that the condensation reaction did not fully proceed during the curing process at $120^{\circ} \mathrm{C}$. To the contrary, the observed decrease in the corresponding peak around $3200 \mathrm{~cm}^{-1}$ (Fig. 4, curve c) may suggest that the desirable condensation proceeds during the curing process at $150{ }^{\circ} \mathrm{C}$.

Figure 5 shows FTIR spectra of polySQ-QAS membranes, obtained via curing process at $150{ }^{\circ} \mathrm{C}$ for $3 \mathrm{~h}$, before and after being soaked in methanol for $24 \mathrm{~h}$.

From the observation that the N-C deformation peak arising from QAS at $1465 \mathrm{~cm}^{-1}$ is present after being soaked in methanol, it is postulated that QAS is fully sustained in polySQ membrane without leakage. While the polySQ-QAS membrane cured at $120^{\circ} \mathrm{C}$ had a great number of fine cracks and broke to pieces, after being soaked in methanol. It was, therefore, difficult to perform a similar evaluation of the preservation of QAS in the membrane state before and after being soaked in methanol.

In conclusions, based on a detachable FET gate metal

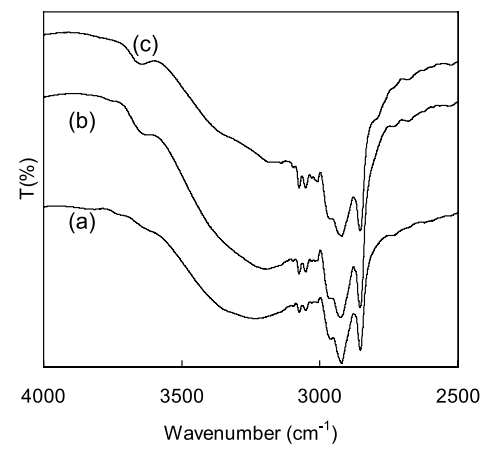

Fig. 4 FTIR spectra of polySQ-QAS membranes. (a) no curing, (b) cured at $120{ }^{\circ} \mathrm{C}$ for $3 \mathrm{~h}$ and (c) cured at $150{ }^{\circ} \mathrm{C}$ for $3 \mathrm{~h}$.

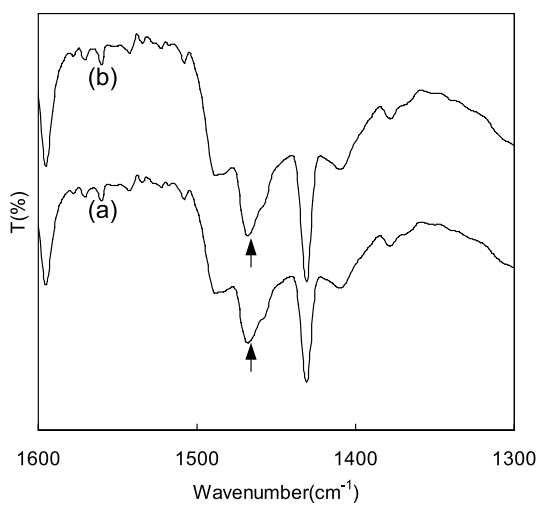

Fig. 5 FTIR spectra of polySQ-QAS membranes, cured at $150{ }^{\circ} \mathrm{C}$ for 3 h. Curves (a) and (b), before and after being soaked in methanol for $24 \mathrm{~h}$.

disk structure, curing of polySQ membrane at relatively high temperatures became possible. Such a detachable sensing head structure was beneficial to obtain the durable ion-sensing polySQ membrane coated ISFET. The results obtained in this study are expected to be applicable to the preparation of various ISFETs with different ion-sensing polySQ membranes.

\section{Acknowledgement}

Y. T. (Domestic Research Fellow) was financially supported by Japan Science and Technology Corporation.

\section{References}

1) R. H. Baney, M. Itoh, A. Sakakibara, and T. Suzuki, Silsesquioxanes, Chem. Rev., 95, 1409 (1995).

2) Y. Tsujimura, M. Yamane, and S. Wakida, Anal. Sci., 17, 485 (2001).

3) A. J. Burzynski and C. A. Johnson, U.S. Patent, 3451838 (1969).

4) A. J. Burzynski, U.S. Patent, 3460980 (1969).

5) H. Schmidt, H. Scholze, and A. Kaiser, J. Non-Crystalline Solids., 63, 1 (1984).

6) G. Orcel and L. Hench, J. Non-Crystalline Solids, 79, 177 (1986).

7) K. Nagy and T. A. Fjeldly, Talanta, 26, 811 (1979).

8) M. Thompson, U. J. Krull, and P. J. Worsfold, Talanta, 26, 1015 (1979).

9) C. W. Davies, J. Chem. Soc., 2093 (1938). 